\title{
Migraine in Nursing Students-A Study from a Tertiary Care Center in South India
}

\author{
Bindu Menon ${ }^{1}$ Nikethana Remadevi ${ }^{2}$ \\ ${ }^{1}$ Department of Neurology, Apollo Specialty Hospitals, Nellore, \\ Andhra Pradesh, India \\ ${ }^{2}$ Department of Nursing, Apollo Speciality Hospitals, Nellore, \\ Andhra Pradesh, India
}

\begin{abstract}
Address for correspondence Bindu Menon, MD, DM, DNB, PGDCN FRCP, MNAMS, FICP, FIAN, Department of Neurology, Apollo Speciality Hospitals, Nellore, Andhra Pradesh 524004, India (e-mail: bindu.epilepsycare@gmail.com).
\end{abstract}

\begin{abstract}
Keywords

- migraine

- nurses

- triggers

Objective Nursing profession is subject to occupational stress, which can be a trigger for headaches. Our study aimed to study the prevalence of migraine, its characteristics, triggers, and relieving factors among nursing students in a tertiary care center. Materials and Methods This study was performed in a super-specialty hospital in South India. A structured questionnaire captured data on the occurrence of headache, demographics, aura, triggering factors, relieving factors, and lifestyle habits. Results are presented in numbers and percentage.

Results A total of $20 \%$ of nursing students in the study had headache of which $85 \%$ had migraine. Weekly and daily attacks were reported in 12 and $4 \%$ students, respectively. Twenty-two percent had headache severity of more than 5 visual analogue scale. Most common accompanying symptoms were photophobia (80\%), phonophobia (70\%), nausea (75\%), vomiting (71\%), neck pain (25\%), and vertigo (20\%). Thirty-nine percent had auras. Ninety-five percent reported triggers with $70 \%$ students having more than one trigger. Sleep was the relieving factor in $69 \%$, head massage in $50 \%$, and relaxing from work in $48 \%$.

Conclusion The most common type of primary headache in nurses in our study was migraine. More than three-fourths nurses reported triggers and relieving factors. Addressing these factors could help in managing migraines and help in improving the quality of life and increased work productivity of nurses.
\end{abstract}

\section{Introduction}

Headache is a common neurological disorder with significant economic and psychosocial impact on affected individuals. There is dearth of data on the frequency and clinical features in patients with primary headache compared with other neurological disorders. Of note, the uncertain nature of this disease leads to frequent absenteeism and decreased productivity at work.

Nursing profession is subject to occupational stress, which can be a trigger for headaches. These stressors include, long working hours, erratic food habits, and lack of sleep among several others. It has been reported in a study that stress was common among health care professionals and physicians and nurses were more vulnerable to stress. ${ }^{1}$ Others have shown that prolonged work hours, working more than 8 hours at a stretch can trigger migraine. ${ }^{2}$ Further, frequent absenteeism due to headache resulted in decreased work efficiency and decreased productivity. ${ }^{3}$ Our study aimed to determine the prevalence of migraine among nursing students in a tertiary care center. Additionally, we examined the migraine characteristics, triggers, and relieving factors.
DOI https://doi.org/ 10.1055/s-0040-1721556 ISSN 0976-3147.

\footnotetext{
(c) 2021. Association for Helping Neurosurgical Sick People.

This is an open access article published by Thieme under the terms of the Creative Commons Attribution-NonDerivative-NonCommercial-License, permitting copying and reproduction so long as the original work is given appropriate credit. Contents may not be used for commercial purposes, or adapted, remixed, transformed or built upon. (https://creativecommons.org/licenses/by-nc-nd/4.0/) Thieme Medical and Scientific Publishers Pvt. Ltd., A-12, 2nd Floor, Sector 2, Noida-201301 UP, India
} 


\section{Materials and Methods}

This study was performed in a super-specialty hospital in South India. The study population included nursing students. Students who confirmed to have headaches in the last 1 year were included in the study. The purpose of the study was explained to the participants by the first author. A structured questionnaire was administered to consented students and the completed ones were collected on the spot. The questionnaire captured data on the occurrence of headache, demographics, aura, triggering factors, relieving factors, and lifestyle habits. Pain intensity was assessed by the visual analogue scale (VAS). The headache diagnosis was made according to the International Headache Society criteria. ${ }^{4}$

Nurses who were unwilling to participate or had secondary headache were excluded from the study. Data was tabulated and analyzed. Results are expressed in numbers and percentage. The Institutional Ethical Committee approved the study.

\section{Results}

Seven hundred and fifty students were included in the study. All were females. A total of 172 students confirmed of having headache and filled the questionnaire. Twenty-two students were excluded from the study as the questionnaire was not completely filled and an inference could not be made from the insufficient data. Of the 150 students, 127 (85\%) students fulfilled the criteria of migraine according to the IHS criteria. The rest (15\%) had other forms of headaches.

Headache was reported in $20 \%$ of the total-nursing students and of which $85 \%$ had migraine. The attacks were more than one per month in $46 \%$ of students and once in 3 months in $38 \%$, whereas weekly and daily attacks were reported in 12 and $4 \%$ students, respectively.

Severity of headache was more than 5 VAS in $22 \%$ of the students. None had pain severity of VAS 10 . However, $24 \%$ students went to emergency services during their episodes of headache. Seventeen percent of the total sample reported motion sickness in their childhood. Accompanying symptoms were common (-Table 1). Most common accompanying symptoms were photophobia (80\%), phonophobia (70\%), nausea (75\%), and vomiting (71\%). Neck pain in $25 \%$ and vertigo in $20 \%$ were also common accompaniments. Auras were seen in $39 \%$ of the students in the form of visual-flickering lights, dazzling zigzag, tunnel like vision, objects looking small, or big and sensory auras like pins and needles. Triggers were identified in $95 \%$ with more than one trigger in $70 \%$ students ( - Table 2). Sleep was the relieving factor in $69 \%$, head massage in $50 \%$, and relaxing from work in $48 \%$. Thirteen percent had relief of pain after vomiting.

Twenty one percent of students had more than three cups of coffee/tea in a day. However, none had commented about it being a relieving factor. Sixty-one percent of the students slept more than 7 hours; however, the sleep timings were dependent upon their shiftwork. Twenty percent found time for workout, which was mainly walking. Thirty-five percent skipped meals due to headache. Phone use in the form of calls
Table 1 Accompanying symptoms of headache

\begin{tabular}{|l|l|l|}
\hline No. & Description & $\%$ \\
\hline 1 & Photophobia & 80 \\
\hline 2 & Nausea & 75 \\
\hline 3 & Vomiting & 71 \\
\hline 4 & Phonophobia & 70 \\
\hline 5 & Neck pain & 25 \\
\hline 6 & Vertigo & 20 \\
\hline 7 & Weakness in face/arm/leg & 8 \\
\hline 8 & Drooping eye lid & 7 \\
\hline 9 & Numbness in face/arm/leg & 7 \\
\hline 10 & Visual disturbances & 5 \\
\hline 11 & Diarrhea & 6 \\
\hline 12 & Speech disturbance & 5 \\
\hline 13 & Diarrhea & 4 \\
\hline
\end{tabular}

Table 2 Triggering factors of headache

\begin{tabular}{|l|l|l|}
\hline No & Descriptions & $\%$ \\
\hline 1 & Loud noise and bright light & 79 \\
\hline 2 & Strong smell & 77 \\
\hline 3 & Mental stress & 62 \\
\hline 4 & Prolonged computer use & 50 \\
\hline 5 & Missing food & 44 \\
\hline 6 & Excess sleep or lack of sleep & 31 \\
\hline 7 & Excess use of mobile phone & 24 \\
\hline 8 & Menstrual periods & 18 \\
\hline 9 & Journey & 14 \\
\hline 10 & Coffee & 7 \\
\hline 11 & Cool drinks & 6 \\
\hline 12 & Head movement & 8 \\
\hline 13 & Warm weather & 8 \\
\hline 14 & Head bath & 6 \\
\hline
\end{tabular}

was high and $62 \%$ spend more than half an hour on phone calls. Analgesic use as over the counter medication was limited and seen in only $9 \%$. Fifty percent had consulted a neurologist for their headache. Sixty-two percent attributed their headaches to stressful lifestyle. Absenteeism from work was seen in only $9 \%$.

\section{Discussion}

Our study showed that $20 \%$ of the nursing students had headache. A 5-year population-based study conducted in Taiwan investigating the risk of migraine in health care professionals noted that incidence rates of migraine were $3.28 \%{ }^{5}$ And among the health care professionals, nurses had higher migraine risk than physicians. ${ }^{5,6}$ Migraine is a disabling disease ranking sixth as measured by the years lived with disability worldwide. ${ }^{7}$ Primary headaches are common and lead to significant disability and distress. This could lead to frequent absenteeism or reduced efficiency of work. Our study 
population had more migraine without aura (61\%) than with aura (39\%). Auras can be disabling and can restrict activity of the individual. Visual auras were more common and varied in the form of flickering lights, dazzling zigzag lines, tunnel like vision, micropsia, and macropsia. The details of the aura were, however, not enquired, as this was a questionnaire-based study. Visual auras have been noted to be more common than the sensory auras in other studies. ${ }^{8}$ One-fourth of the students had headache severity more than 5 VAS and needed emergency help. We did not include the MIDAS questionnaire as the time taken to answer the questions are long and would require guidance for completion.

Neck pain as an accompanying symptom has been noted in tension type or cervicogenic headache in the past. However, it is increasingly being recognized as an accompanying symptom of migraine as well. ${ }^{9}$ Migraine and vertigo are frequent accompaniments. They could be as chance alone, comorbid disease or vestibular migraine. However, this conclusion could not be made from the questionnaire. Twenty-five percent of the patients with migraine have been noted to have vertigo. ${ }^{10} \mathrm{~A}$ study had also documented migraine being the most common cause of vertigo. ${ }^{11}$

Several intrinsic and extrinsic factors can trigger migraine and these identifiable triggers have therapeutic implications in reducing the migraine attacks. The most common trigger recalled was bright light sound and strong smells in our study. Light as a possible migraine trigger has been identified in previous studies. ${ }^{12}$ A larger study from northern Norway found that almost $12 \%$ of migraineurs reported increase in migraine headaches during midnight-sun season indicating the susceptibility of light on migraine. ${ }^{13}$ Sensitivity to odors is also commonly noted in some migraineurs. Most commonly identified odors are perfume, cigarette smoke, and cleaning products. ${ }^{14}$ Cleaning products could be a possible trigger in the hospital setting; however, any strong odor could be a trigger. Moreover, particular hospital areas like casualty and operating theater where noise and odor are different could be the trigger in certain students. However, increased sensitivity to smell, light, and sounds is also common during the migraine attack and should be borne in mind while treating patients with migraine. With digitalization of hospital records, computer use has increased for entering data. We found computer use as a migraine risk in around $50 \%$ of the subjects and phone in one-fourth of the subjects. The use of phone has increased globally. Studies have shown that increasing use of mobile phones has been linked to increased headache. ${ }^{15}$ The effect of electromagnetic fields from mobile phones has always been a source of concern. A study conducted on 2,150 high school students found an association between mobile phones and headache along with concentration difficulties, fatigue, sleep disturbances, and warming of the ear. The study also noted a relationship between frequency of headache and calls made a day. ${ }^{16}$ In our study, $62 \%$ of the students spend more than half an hour on phone calls. In an earlier study, phone use was also a factor in chronic headaches. ${ }^{17}$

Mental stress and lack or excess sleep were other identified triggers in our study. Heavy workloads, shift work, and sleep disturbance are commonly recognized in health care professionals. Interestingly, our study found that increased sleeping hours was an equal trigger factor for migraine. In fact, $61 \%$ of students had slept for more than 7 hours in a day; however, the sleep timings were depending on their shifts. The reason could be cumulative sleep debt during night shifts and later increased sleep as compensatory mechanism. However, studies have shown that such sleep patterns do not help reducing headaches. ${ }^{18}$

Missing meals and fasting have long been reported as headache triggers and were seen in $44 \%$ of our study subjects. This could be easily addressed by encouraging them small frequent healthy snacks during working hours. Recognizing trigger factors can help in giving individualized recommendations for persons suffering from migraine. Recognition of the trigger factors could be important factor for the lesser frequency of migraine in our study group. Weekly migraine attacks were seen in $12 \%$ and daily in $4 \%$. Relieving factors are equally important to reduce the migraine burden. Relieving factors like good sleep, head massage, or relaxing from work point out the work stress as an important trigger factor. Sleep and relaxation are identified relieving factors, however, 50\% also reported head massage, which is relatively less reported. Head massage was found a reliving factor in yet another study. ${ }^{19}$

Identification of nonpharmacological relieving factors is as important as recognition of the trigger factors. This will also help in reducing the analgesic use as we found in our study where analgesic usage was only $9 \%$.

Lifestyle modification forms yet another method for preventing migraine attacks. We enquired about the lifestyle of the students. Surprisingly, the sleep hours were adequate in more than $50 \%$ of the students, however, the timings were erratic according to their duties. Exercises in any form were undertaken by less than one-fourth of the students. Studies have shown that higher physical activity levels lead to lower migraine frequency leading to less migraine-related disability. ${ }^{20}$ Skipping meals was very common and is a known migraine trigger. The trigger factors and the relieving factors portray the lifestyle changes, which should be made in the study group.

There are limitations in this study. First, it was not an ageand sex-matched study, as all the subjects in the study were females of the same age group. As we used a questionnaire asking about their previous experiences related to migraine, it might have produced a recall bias. However, we tried to minimize this by ensuing that the checklist was comprehensive and multifactorial.

\section{Conclusion}

Our study showed that migraine was the most common type of primary headache in nurses. There were several identifiable triggers and also relieving factors. Addressing these factors could help in managing migraines and help in improving the quality of life and increased work productivity of nurses. 


\section{Ethical Approval}

The Institutional Ethical Committee approved the study.

\section{Funding}

None.

\section{Conflict of Interest}

None declared.

\section{References}

1 Wilkins K. Work stress among health care providers. Health Rep 2007;18(4):33-36

2 Wang Y, Xie J, Yang F, et al. The prevalence of primary headache disorders and their associated factors among nursing staff in North China. J Headache Pain 2015;16:4

3 Selekler MH, Gökmen G, Steiner TJ. Productivity impact of headache on a heavy-manufacturing workforce in Turkey. J Headache Pain 2013;14:88

4 Headache Classification Committee of the International Headache Society (IHS). The International Classification of Headache Disorders, 3rd edition. Cephalalgia 2018;38(1):1-211

5 Kuo WY, Huang CC, Weng SF, et al. Higher migraine risk in healthcare professionals than in general population: a nationwide population-based cohort study in Taiwan. J Headache Pain 2015;16:102

6 Xie W, Li R, He M, et al. Prevalence and risk factors associated with headache amongst medical staff in South China. J Headache Pain 2020;21(1):5

7 Steiner TJ, Stovner LJ, Vos T. GBD 2015: migraine is the third cause of disability in under 50s.J Headache Pain 2016;17(1):104

8 Viana M, Sances G, Linde M, et al. Clinical features of migraine aura: Results from a prospective diary-aided study. Cephalalgia 2017;37(10):979-989
9 Pradhan S, Choudhury SS. Clinical characterization of neck pain in migraine. Neurol India 2018;66(2):377-384

10 Lempert T, Neuhauser H. Epidemiology of vertigo, migraine and vestibular migraine. J Neurol 2009;256(3):333-338

11 Vuković V, Plavec D, Galinović I, Lovrencić-Huzjan A, Budisić M, Demarin V. Prevalence of vertigo, dizziness, and migrainous vertigo in patients with migraine. Headache 2007;47(10): 1427-1435

12 Hauge AW, Kirchmann M, Olesen J. Trigger factors in migraine with aura. Cephalalgia 2010;30(3):346-353

13 Salvesen R, Bekkelund SI. Migraine, as compared to other headaches, is worse during midnight-sun summer than during polar night. A questionnaire study in an Arctic population. Headache 2000;40(10):824-829

14 Lima AM, Sapienza GB, Giraud VdeO, Fragoso YD. Odors as triggering and worsening factors for migraine in men. Arq Neuropsiquiatr 2011;69(2B) :324-327

15 Wang J, Su H, Xie W, Yu S. Mobile phone use and the risk of headache: a systematic review and meta-analysis of cross-sectional studies. Sci Rep 2017;7(1):12595

16 Durusoy R, Hassoy H, Özkurt A, Karababa AO. Mobile phone use, school electromagnetic field levels and related symptoms: a cross-sectional survey among 2150 high school students in Izmir. Environ Health 2017;16(1):51

17 Khan MM. Adverse effects of excessive mobile phone use. Int J Occup Med Environ Health 2008;21(4):289-293

18 Basner M, Rao H, Goel N, Dinges DF. Sleep deprivation and neurobehavioral dynamics. Curr Opin Neurobiol 2013; 23(5):854-863

19 Haque B, Rahman KM, Hoque A, et al. Precipitating and relieving factors of migraine versus tension type headache. BMC Neurol 2012;12:82

20 Varkey E, Cider A, Carlsson J, Linde M. Exercise as migraine prophylaxis: a randomized study using relaxation and topiramate as controls. Cephalalgia 2011;31(14):1428-1438 